demonstrated high levels of schizophrenia spectrum pathology in Klinefelter (van Rijn et al., 2006a). Difficulties in social adaptation and abnormal brain asymmetries suggest that a genetic mechanism (involving genes on the X chromosome) might affect the development of social cognition in XXY men. Language and emotion are important aspects of social cognition. Reduced or abnormal lateralization of language has been found in Klinefelter's syndrome (Geschwind, 1998; van Rijn et al., 2008). Additionally, impairments in emotional prosody perception have been reported (van Rijn et al., 2007). We expected that lateralization of emotional prosody differed in Klinefelter's syndrome. Therefore, we used fMRI-guided transcranial magnetic stimulation (TMS) to study lateralization effects.

Methods: Seven men with Klinefelter's syndrome and seven controls (matched on age, gender and education) performed an emotional prosody task during fMRI-scanning. In this task, subjects attended to the affective (happy, sad, angry or anxious) intonation and had to ignore the neutral semantic content of the sentences. We used fMRIguided TMS (with BrainVoyager neuronavigation) to reduce excitability of areas that were active during emotional prosody processing in the MR scanner (left and right superior temporal gyrus). TMS was performed for 20 minutes on $1 \mathrm{~Hz}$. After stimulation, the subjects performed the emotional prosody task again. In addition, a baseline measurement without TMS was included. Results were analyzed with repeated measures analysis of variance (ANOVA).

Results: Klinefelter patients showed a trend to react slower than controls $(p=0.12)$. They also showed longer reaction times on the angry condition after stimulation of the right superior gyrus $(p=0.05)$, whereas controls reacted faster. No other effects of TMS reached significance.

Discussion: Men with Klinefelter's syndrome responded more slowly to sentences with an angry intonation in the emotional prosody task after right STG stimulation, whereas controls responded faster. The emotion angry is hypothesized to be primarily processed by the left hemisphere. Thus, the involvement of the right STG in anger prosody processing in Klinefelter patients suggests a switch in lateralization. The faster responses in the healthy group for this emotion might be due to callosal disinhibition of the left hemishere by the right hemisphere TMS. As fMRI studies have suggested similar patterns of aberrant lateralization in Klinefelter syndrome and schizophrenia, our results may have implications for understanding schizophrenia.

doi:10.1016/j.schres.2010.02.347

\section{Poster 120}

\section{CORTICAL DYSFUNCTION IN ADOLESCENTS WITH SCHIZOPHRENIA DURING WORIING MEMORY COMPONENT PROCESSES - A FUNCTIONAL MAGNETIC RESONANCE IMAGING STUDY}

Robert A. Bittner ${ }^{1,3}$, Corinna Haenschel ${ }^{1,3,5}$, Alard Roebroek ${ }^{4}$, Fabian Haertling $^{2}$, Anna Rotarska-Jagiela ${ }^{3}$, Konrad Maurer ${ }^{1}$, Rainer Goebel ${ }^{4}$, Wolf Singer ${ }^{3}$, David E.J. Linden ${ }^{5}$

${ }^{1}$ Laboratory for Neurophysiology and Neuroimaging, Department of Psychiatry, Goethe-University Frankfurt am Main Germany; ${ }^{2}$ Department of Child and Adolescent Psychiatry, Goethe-University Frankfurt am Main Germany; ${ }^{3}$ Department of Neurophysiology, Max-Planck-Institute for Brain Research Frankfurt am Main Germany; ${ }^{4}$ Department of Cognitive Neuroscience, Faculty of Psychology, Maastricht University Maastricht Netherlands; ${ }^{5}$ Wolfson Centre of Clinical and Cognitive Neuroscience, School of Psychology, University of Wales, Bangor Bangor Wales

Background: Working memory (WM) impairment in schizophrenia (SCZ) is caused by deficits of both encoding and maintenance processes. Prefrontal cortical dysfunction has also consistently been implicated. Yet, results from functional magnetic resonance imaging (fMRI) studies have been contradictory with reports of both decreased and increased prefrontal cortical activation. This discrepancy has been explained by models of a dynamic WM load dependent dysfunction of prefrontal cortex. Abnormal functional connectivity also seems to contribute to WM deficits. However, the use of blocked experimental designs - often in conjunction with the n-back task - has prevented the isolation of WM component processes in the majority of fMRI studies. Therefore, the relationship between impairments in specific WM component processes with abnormal with abnormal cortical activation, connectivity and WM load sensitivity in SCZ is still poorly understood.

Methods: We used event-related fMRI to examine differences in brain activation and functional connectivity during the encoding, maintenance and retrieval stages of a visual WM task in 17 adolescents with early-onset SCZ (age 15 to 20 years) and 17 matched controls. Up to three abstract visual shapes had to be maintained for 12 seconds before comparing them to a test stimulus. Results: Patients had reduced WM capacity, which was related to lower activation in left ventrolateral prefrontal cortex (VLPFC) and extrastriate visual cortex during encoding. During early maintenance patients showed a switch from hyper- to hypoactivation with increasing WM load in a fronto-parietal network which included left dorsolateral prefrontal cortex (DLPFC). Furthermore, abnormally increased suppression of default mode areas by patients was correlated with WM capacity during late maintenance. During retrieval right inferior VLPFC hyperactivation was correlated with encoding-related hypoactivation of left VLPFC in patients. Cortical dysfunction in patients during encoding and retrieval was accompanied by abnormal functional connectivity between fronto-parietal and visual areas.

Discussion: Patients showed a primary encoding deficit independent of WM load duet to a dysfunction of VLPFC and visual areas. Prefrontal hyperactivation during retrieval seems to be a secondary consequence of this deficit. In contrast, WM load dependent fronto-parietal dysfunction and dysregulation of default mode areas seem to contribute to impaired WM maintenance. These results indicate that WM dysfunction in SCZ is the result of a combination of disturbances in distinct cortical networks which support the individual WM component processes. In contrast, WM load dependent abnormalities seem to be confined primarily to early maintenance. A separate analysis of WM component processes and parametric manipulation of WM load may help to develop more valid and reliable intermediate phenotypes for genetic studies. Focusing on impairments of specific component processes could also lead to more targeted and effective psychological and pharmacological cognitive remediation strategies.

doi:10.1016/j.schres.2010.02.348

\section{Poster 121 \\ ALTERATIONS IN FUNCTIONAL BRAIN NETWORK STRUCTURE INDUCED BY SUBCHRONIC PHENCYCLIDINE (PCP) TREATMENT PARALLEL THOSE SEEN IN SCHIZOPHRENIA}

$\underline{\text { Neil Dawson }}^{1}$, Des Higham ${ }^{2}$, Judith Pratt ${ }^{1}$, Brian Morris ${ }^{1}$

${ }^{1}$ PsyRING, Universities of Glasgow and Strathclyde Glasgow, Lanarkshire, United Kingdom; ${ }^{2}$ University of Strathclyde Glasgow, Lanarkshire, United Kingdom

Background: Quantitative analysis of complex network structure, based on graph theory, has recently been applied to elucidate the organisation of structural and functional brain networks (Bullmore and Sporns, 2009) in both healthy humans and in disease states, including schizophrenia (Bassett et al., 2008; Liu et al., 2008). These methods are yet to be applied to brain imaging data from preclinical models relevant to mental health. Here we investigate network 
structure in functional brain networks in a preclinical model relevant to schizophrenia (Pratt et al., 2008).

Methods: Cerebral metabolism in 64 brain regions was determined in control rats (saline, male, Long-Evans, $\mathrm{n}=7$ ) and rats treated subchronically with PCP (2.58 mg.kg ${ }^{-1}$, i.p, $1 \mathrm{x}$ daily for 5 days, $\mathrm{n}=9$ ) by semi-quantitative 2-deoxyglucose (2-DG) autoradiography (Dawson et al., 2009). Overt alterations in metabolism between groups were analysed by t-test. For graph theoretical analysis binary adjacency matrices, over a range of correlation thresholds (Pearson's co-efficient 0.4 to 0.5 ), were generated from the brain region partial correlation matrix of each experimental group. Global network architecture was characterised in terms of the mean degree, average path length, mean clustering coefficient and small-worldness at each threshold. In addition, centrality analysis (degree, betweeness and closeness) was used to identify hub regions in these networks. Smallworld properties and hub region identification were determined by statistically comparing real with calibrated random (Erdös-Rényi) graphs. Statistical differences in network architecture between groups was analysed using repeated measures ANOVA. Alterations in centrality measures between groups were analysed by comparing regional z-scores, generated relative to random networks, using t-test with Bonferoni correction. Significance was set at $\mathrm{p}<0.05$.

Results: Subchronic PCP-treatment induced overt hypometabolism in select prefrontal and thalamic regions in accordance with previous reports (Pratt et al., 2008). The functional brain network in PCP-treated animals had a significantly reduced mean degree, increased average path length and reduced mean clustering in comparison to that in controls. The network in PCP-treated animals also displayed a significantly higher small-worldness than that in controls. In control animals the mediodorsal and reticular (dorsal and ventral) thalamus and locus coeruleus were identified as important hubs, across all centrality measures. Of these regions the reticular thalamus (ventral and dorsal) and locus coeruleus lost their hub status in PCP-treated animals. Several additional thalamic regions (anteromedial, centrolateral, centromedial and the nucleus reuniens) also showed reduced centrality in PCP-treated animals.

Discussion: This study is the first to apply the quantitative analysis of network structure to functional brain imaging data from a preclinical model relevant to schizophrenia. The global architecture of this network in control animals, including a small-world topography, is consistent with reports from human studies. In addition, PCPinduced alterations in network structure parallel functional network alterations seen in schizophrenia (Liu et al., 2008). Furthermore, these results provide new insight into alterations in functional brain networks which may contribute to the overt alterations in cerebral metabolism seen in schizophrenia. Bassett et al, 2008. J.Neurosci. 28:9239 Bullmore and Sporns, 2008. Nat.Rev. Neurosci. 10:186 Dawson et al, 2009 J.Neuro.Res. 87:2375 Liu et al, 2008 Brain 131:945 Pratt et al, 2008 Br.J.Pharmacol. 153:S465.

doi:10.1016/j.schres.2010.02.349

\section{Poster 122}

BRAIN ACTIVITY DURING SOCIAL COGNITION TASIKS IN INDIVIDUALS WITH SCHIZOPHRENIA, THEIR UNAFFECTED SIBLINGS, AND HEALTHY CONTROLS

Salvador M. Guinjoan ${ }^{1,2}$, Delfina de Achaval $^{1,2}$, Mirta Villarreal ${ }^{1,2}$, Elsa Y. Costanzo ${ }^{1}$, Rocio Berhongaray ${ }^{1}$, Jazmin Douer ${ }^{1}$, Julieta Lopez ${ }^{1}$, Martina C. Mora ${ }^{1}$, Rodolfo Fahrer ${ }^{1}$, Ramon C. Leiguarda ${ }^{1}$, Salvador M. Guinjoan ${ }^{1,2}$

${ }^{1}$ Fundación para la Lucha contra las Enfermedades Neurológicas de la Infancia (FLENI) Capital Federal, Buenos Aires, Argentina; ${ }^{2}$ Consejo Nacional de Investigaciones Científicas y Técnicas (CONICET) Capital Federal, Buenos Aires, Argentina
Background: Several studies have shown that patients with schizophrenia have impaired performance in various aspects of social cognition including emotion processing, theory of mind, and moral judgment. Most of the neuroimaging studies have compared patients and healthy controls during such mental activity because the understanding of the neural basis of social cognition might help to explain some deficits in social functioning in this group of patients. The present study examined brain activation patterns during social cognition tasks in patients with schizophrenia, and try to determine whether alterations in social cognition reflect a trait that can be detected in non-psychotic relatives of patients with schizophrenia.

Methods: Eight patients with schizophrenia (age $33.9 \pm 13,2$ females), ten non-psychotic relatives (age $32 \pm 3.7,4$ females) and ten matched comparison subjects (age 27.5 $\pm 7,4$ females) underwent BOLD (blood-oxygen-level-dependent) functional magnetic resonance imaging during visual presentation of different social cognition paradigms. Emotion processing was measured by the Ekman Faces Test using a target (basic emotions) and a control (gender) condition. Theory of Mind (ToM) paradigms were focused on the ability to discriminate complex mental states in Faces and Reading the Mind in the Eyes task, with a target (complex mental states) and a control (gender) condition as well. Moral judgment task consisted in 40 short passages, half of them with moral content, in which the subject has to judge the characters actions. Random effects analysis was done for each task within groups, measuring signal changes between the target and control conditions of each paradigm.

Results: The Reading the Mind in the Eyes (ToM task) brought about activation in the left inferior frontal gyrus, near Brodmann's areas 44 and 45 , in all groups. Both patients and their siblings showed activation in the same area of the right hemisphere although with less intensity, and in the left striatum. Patient's siblings showed bilateral activation of the middle occipital gyrus. During a moral judgement task, right inferior frontal gyrus was activated in both patients and their relatives (but more intense in the former), and relatives activated right ventromedial prefrontal cortex as well. In this task, healthy persons activated preferentially bilateral postcentral gyri. Both facial tests evoked much lesser activation in patients than in the other two groups. Healthy subjects activated preferentially bilateral middle frontal gyri. Patients' siblings displayed the most intense activation of all groups including bilateral middle and inferior frontal gyri, parahippocampi, bilateral occipital structures and bilateral cerebellar structures.

Discussion: Reading the Mind in the Eyes and moral judgement tests evoked partially overlapping cerebral activation patterns in patients and their siblings but not in comparable healthy individuals. Emotion processing as assessed by social cognitive tasks involving faces evoked strong cerebral activation in unaffected siblings of schizophrenia patients. The present results suggest that social cognitive and moral tasks are associated with activation of brain areas partially similar in patients with schizophrenia and their unaffected siblings, and distinct from those in healthy individuals.

doi:10.1016/j.schres.2010.02.350

\section{Poster 123 \\ SIMILAR LANGUAGE ACTIVATION IN NON-PSYCHOTIC INDIVIDUALS WITH AUDITORY VERBAL HALLUCINATIONS AND CONTROL SUBJECTS}

Kelly M. Diederen, Antoin D. De Weijer, Kirstin Daalman, Bas F. Neggers, Rene S. Kahn, Iris E. Sommer University Medical Center Utrecht \& Rudolf Magnus Institute for Neuroscience Utrecht, Utrecht, Netherlands

Background: Psychosis is a debilitating syndrome, characterised by hallucinations, delusions and disorganisation. A well-replicated find- 